\title{
A Linear Algorithm for Edge-Face Coloring Series-Parallel Graphs ${ }^{\star}$
}

\author{
Jian-Liang $\mathrm{Wu}^{1}$ and Ping Wang ${ }^{2}$ \\ ${ }^{1}$ Department of Mathematics, Shandong University, Jinan, Shandong, P.R. China \\ jlwu@math.sdu.edu.cn \\ ${ }^{2}$ Dept. of Math., Stats. and Computer Science, St. Francis Xavier University, \\ Antigonish, NS, Canada, B2G 2W5
}

\begin{abstract}
Let $G$ be a series-parallel graph. In this paper, we present a linear algorithm of constructing an oriented binary decomposition tree of $G$. We use it to find 33 unavoidable subgraphs of $G$. Based on these 33 avoidable subgraphs, we can determine the edge-face chromatic number, denoted by $\chi_{e f}(G)$, of $G$ where $G$ is 2-connected and $\Delta(G)=$ 5 . This completes the literature of determining $\chi_{e f}(G)$ for 2-connected series-parallel graphs.
\end{abstract}

Keywords: edge-face coloring, decomposition tree, series-parallel graph.

\section{Introduction}

In this paper all graphs are simple, undirected and plane. A plane graph $G$ is a particular drawing of a planar graph on the Euclidean plane. Let $G$ be a plane graph with vertex set $V(G)$, edge set $E(G)$ and face set $F(G)$. An edge-face coloring of a plane graph $G$ is a coloring of $E(G) \cup F(G)$ such that no two adjacent or incident elements receive the same color. A 2-terminal simple seriesparallel graph, denoted by $S P$-graph, is a graph with two distinguished vertices (the terminals) that is obtained as follows:

Base case A single edge $u v$ is a 2-terminal SP-graph with terminals $u$ and $v$. Recursive step Let $G_{1}$ be a SP-graph with source $u\left(G_{1}\right)$ and terminal $v\left(G_{1}\right)$, and let $G_{2}$ be a SP-graph with source $u\left(G_{2}\right)$ and terminal $v\left(G_{2}\right)$.

-A graph $G$ obtained from $G_{1}$ and $G_{2}$ by identifying $v\left(G_{1}\right)$ and $u\left(G_{2}\right)$ is a SPgraph with source $u(G)=u\left(G_{1}\right)$ and terminal $v(G)=v\left(G_{2}\right)$. Such a connection is called a series connection.

-A graph $G$ obtained from $G_{1}$ and $G_{2}$ by identifying $u\left(G_{1}\right)$ and $u\left(G_{2}\right)$ and $v\left(G_{1}\right)$ and $v\left(G_{2}\right)$ is a SP-graph with source $u(G)=u\left(G_{1}\right)=u\left(G_{2}\right)$ and terminal $v(G)=v\left(G_{2}\right)=v\left(G_{2}\right)$ where $u\left(G_{1}\right) v\left(G_{1}\right)$ and $u\left(G_{2}\right) v\left(G_{2}\right)$ can not be both edges simultaneously in $G_{1}$ and $G_{2}$. Such a connection is called a parallel connection. It is well-known that the following statements are equivalent if $G$ is a 2-connected graph:

^ This research is supported by NSFC $(10471078,10631070,60673059)$ and NSERC. 


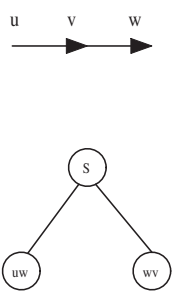

Figure 1-a
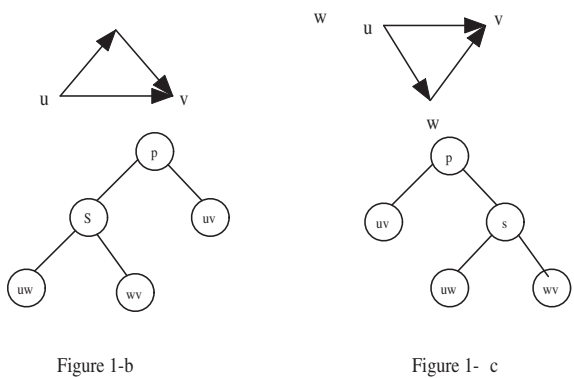

Fig. 1. Examples of series and parallel connections

(1) $\quad G$ does not contain $K_{4}$ as a minor;

(2) $G$ is a 2-terminal SP-graph;

(3) $\quad G$ is a partial 2-tree.

The family of SP-graphs is a distinguished family of plane graphs which is particularly interesting to computer scientists. For example, the problem of determining chromatic index is NP-complete. On the other hand, for plane graphs $\mathrm{G}$, it is not known whether determining $\chi^{\prime}(G)$ is NP-hard. However, there is a linear algorithm to find $\chi^{\prime}(G)$ if $G$ is a series-parallel graph [12. Fast algorithms have been found on the SP-graphs for min-max matching problem, Steiner subgraph and dominating set problems (see [3]). Graph theorists also use SP-graphs in an attempt to solve problems on plane graphs. For example, Seymour's conjecture on chromatic index, if $G$ is a plane graph then $\chi^{\prime}(G)=\max \{\Delta(G),\lceil\Gamma(G)\rceil$ \} , was first proven on outerplane graphs, then on series-parallel graphs (see [5]) where $\Gamma(G)=\max \left\{\frac{2 \bar{X}}{X \mid-1}: X \subset V(G),|X| \geq 3\right.$ and odd $\}$ and $\bar{X}$ denotes the set of edges with both ends in $X$.

In 1975, Mel'nikov 2] conjectured that $\Delta(G) \leq \chi_{e f}(G) \leq \Delta(G)+3$ for any plane graph $G$. Waller [7] (independently by Sanders and Zhao [4) proved the conjecture by using the Four Color Theorem. Wang and Lih [10] recently proved the conjecture without using the Four Color Theorem. Borodin 1 proved that for any plane graph $G, \chi_{e f}(G) \leq \max \{11, \Delta(G)+1\}$. Wang 8 proved that for any outerplane graph $G, \chi_{e f}(G) \leq \max \{7, \Delta(G)+1\}$ and $\chi_{e f}(G)=\Delta(G)$ if $G$ is 2-connected and $\Delta(G) \geq 6$. In [9], Wang and Zhang further improved Wang's result and obtained the edge-face chromatic number of outerplane graphs with $\Delta(G) \geq 5$. Wu and Wang 11 extend the previous result to SP-graphs and proved that $\chi_{e f}(G)=\Delta(G)$ if $G$ is a 2-connected SP-graph with $\Delta(G) \geq 6$. This result provides a better upper bound than the one in Borodin's result and encompasses a larger family of plane graphs than the one given by Wang and Zhang in 9. In the following theorem, we will prove the remaining case where $\Delta(G)=5$. This is the only case which is still open for these types of theorems in which $\chi_{e f}(G)=\Delta(G)$ with $\Delta(G) \geq c$ because of the following example. Let $a b c$ and $x y z$ be two triangles. We form a new graph $G$ by identifying vertices $c$ and $x$ and add a $P_{2}$ between $a$ and $z$. Clearly, $\chi_{e f}(G)=5$ and $\Delta(G)=4$. In 
fact, a family of graphs constructed similarly with any two odd cycles has the same property. This completes the literature of edge-face coloring of 2-connected SP-graphs.

Theorem 1. If $G$ is a 2-connected SP-graph, then $\chi_{e f}(G)=5$ if $\Delta(G)=5$.

\section{Subgraph Identification}

It is well-known that a $\mathrm{SP}$-graph $G$ can be represented by a binary decomposition tree [6]. This technique has been used very successfully to design linear time algorithms to obtain different parameters of SP-graphs (see [12] 13]). In order to apply the binary decomposition tree technique successfully to the face coloring problem, we not only need to construct a binary decomposition tree to represent $G$ but we also require this representation to provide further information about the allocation of faces with respect of its adjacent edges. In the following we shall use the left child and the right child of a node to distinguish whether a face is on the left side or the right side of a particular subgraph. Next we will define an oriented binary decomposition tree of $G$, denoted by $T_{O}$, according to the binary decomposition tree of $G$, denoted by $T$, where $T$ is constructed by the algorithm provided by by Takamizawa et.al. in [6].

Lemma 1. Let $G$ be a 2-terminal SP-graph with terminals $u$ and $v$. Then there exists an acyclic edge orientation of $G$ such that $u$ is the only source and $v$ is the only terminal.

This can be easily showed by using induction to the connection steps in the definition of a 2-terminal simple SP-graph. By Lemma1, we can assign an acyclic edge orientation to $G$ from the source to the sink of $G$. Let $G_{1}$ be a SP-graph with source $u\left(G_{1}\right)$ and terminal $v\left(G_{1}\right)$, and let $G_{2}$ be a SP-graph with source $u\left(G_{2}\right)$ and terminal $v\left(G_{2}\right)$. For any $S$ node in the binary decomposition tree of $T$, we assign $G_{1}$ as its left child and $G_{2}$ as its right child in $T_{O}$ if $v\left(G_{1}\right)=u\left(G_{2}\right)$. For example, Figure 1-a shows a binary decomposition tree which represents a series connection of two oriented edges. For any $P$ node in the binary decomposition tree of $T$, we assign $G_{1}$ as its left child and $G_{2}$ as its right child in $T_{O}$ if $G_{1}$ is on the left side of $G_{2}$ when facing from the source to the terminal in $G_{2}$. Otherwise we assign $G_{2}$ as its the left child and $G_{1}$ as its right child in $T_{O}$ if $G_{1}$ is on the right side of $G_{2}$ when facing from the source to the terminal in $G_{2}$. For example, Figure 1-b and Figure 1-c show two binary decomposition trees which represent two possible parallel connections for the case where $G_{1}=u v$ and $G_{2}=u w v$.

Clearly, all internal nodes are either $S$ or $P$ nodes and all leaves are edges of $G$. We shall use a sequence $S X Y(P X Y)$ to denote a subtree of $T_{O}$ where $X$ and $Y$ are the left child and the right child of $S(P)$ which can be either a subtree or a leaf $(e \in E(G))$. Since we have to color faces of $G, P X Y$ and $P Y X$ might be different. By the symmetry of subgraphs, most of $S X Y$ and $S Y X$ are the same.

Theorem 2. Let $G$ be a 2-connected SP-graph. Then $G$ must contain at least one of the following 33 unavoidable subgraphs (see Figure 2). 

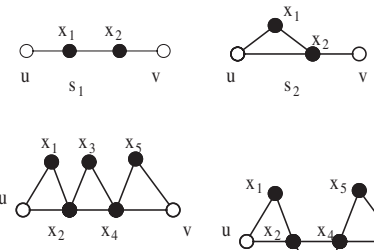

$\int_{\mathrm{s}_{3}}^{\mathrm{x}_{3}} \mathrm{O}^{\mathrm{v}}$

$\overbrace{\mathrm{s}_{4}}^{\mathrm{x}_{2}}$
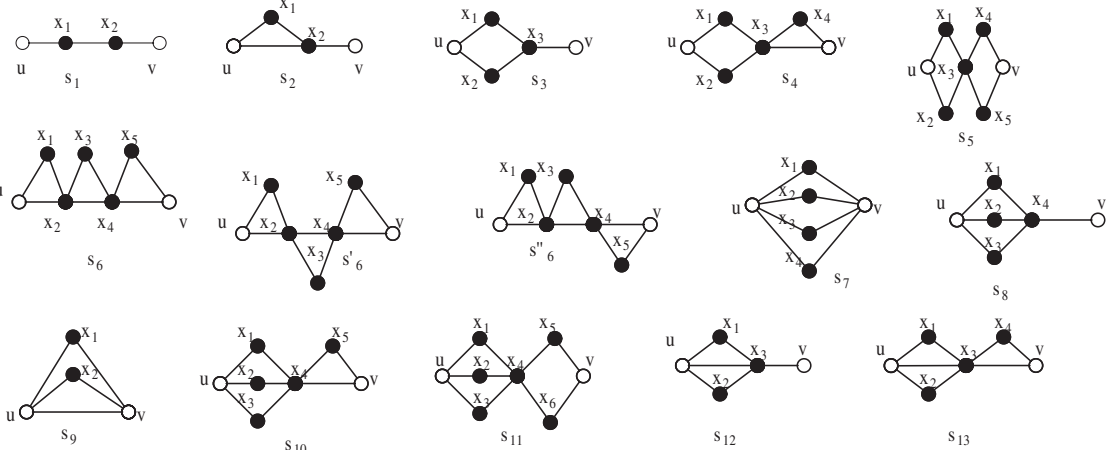

$\overbrace{2}^{x_{1}}$
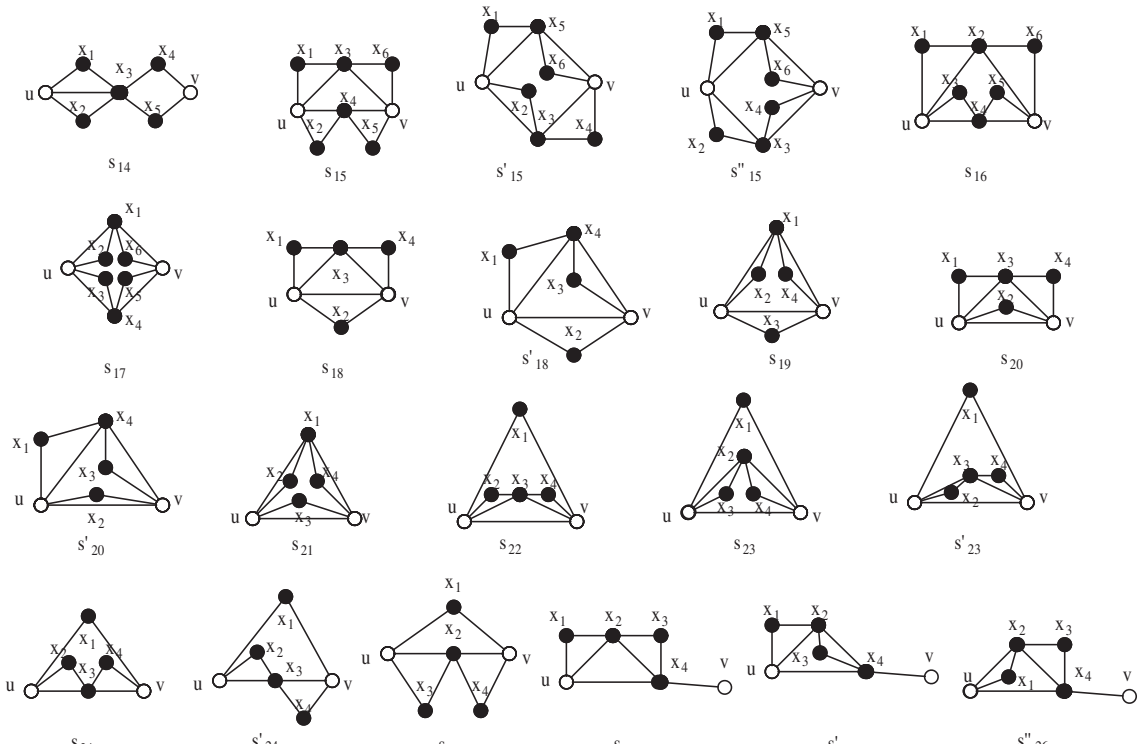

$\mathrm{s}_{24}$
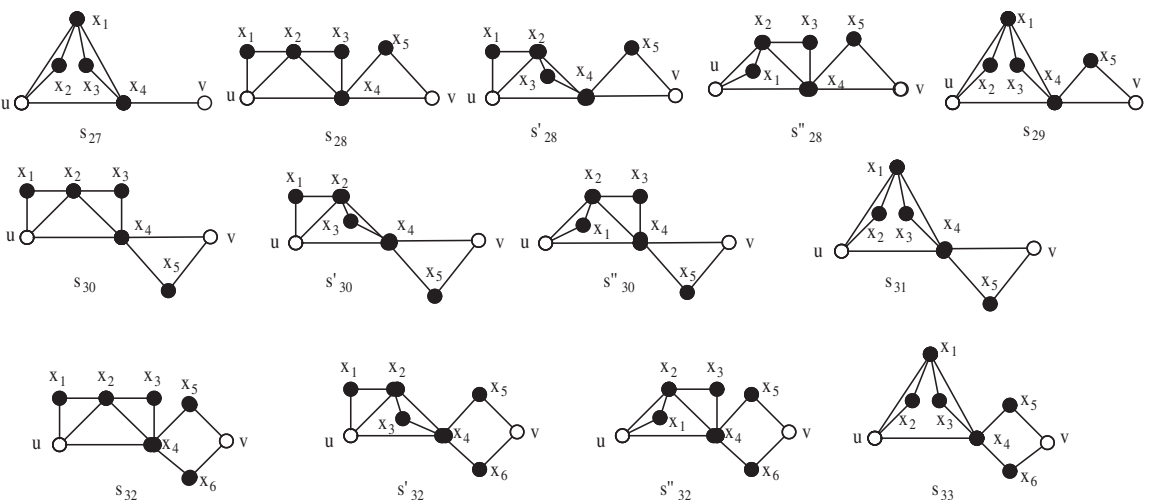

Fig. 2. 33 unavoidable subgraphs 
Proof. Let $P\left\{t_{k}, t_{k-1}, \ldots, t_{1}, t_{0}\right\}$ be the longest path in $T_{O}$ where $t_{0}=e$ is a leaf node and $t_{k}$ is the root node. Since $G$ is a simple graph, the last internal node $t_{1}$ must be a $S$ node. For convenience, let $A_{0}=S e e$ (see Figure 3 ). In the following, we shall move up on the longest path $P$ and find all the desired subgraphs. Since the nodes $t_{0}$ and $t_{1}$ have been discussed above, we shall start from $t_{i}$ where $i \geq 2$ in the following cases.

Case $1 . \quad i=2$

If $t_{2}=S$, then one of its two children of $t_{2}$ must be $A_{0}$ and the other can be either $A_{0}$ or a leaf node. In all three cases, $s_{1}$ is found. If $t_{2}=P$, then there are three possible combinations and we call the three resulting subgraphs $A_{1}=P A_{0} e, A_{1}^{\prime}=P e A_{0}$ and $A_{2}=P A_{0} A_{0}$ (see Figure 3 ).

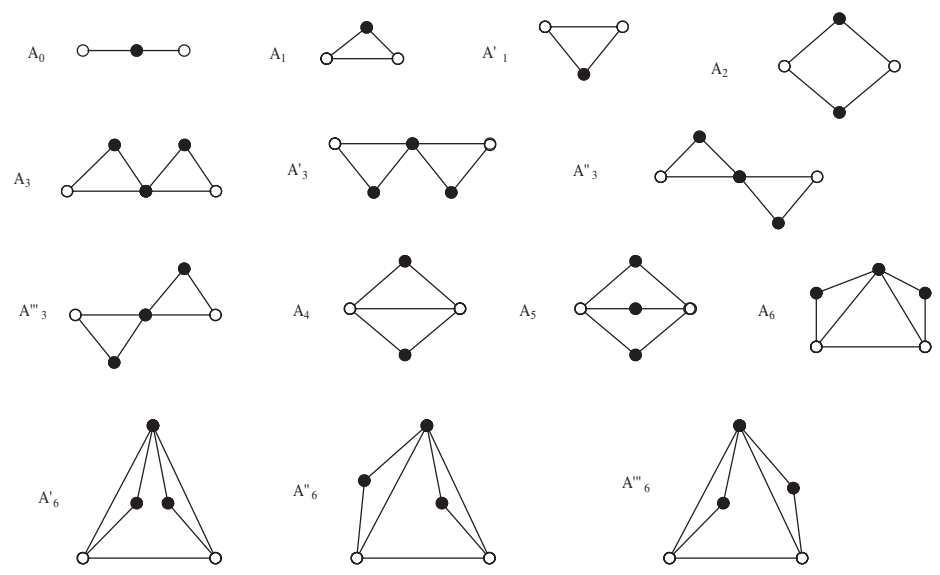

Fig. 3. $A_{i}$ for $i=1,2, \ldots, 6$

Case 2. $\quad i=3$

One of the children of $t_{3}$ must be in $\left\{A_{1}, A_{1}^{\prime}, A_{2}\right\}$.

Subcase 2.1. $\quad t_{3}=S$

$S A_{1} A_{2}$ and $S A_{1}^{\prime} A_{2}$ leads $s_{4} \subseteq G$. $S A_{1} e$ leads to $s_{2} \subseteq G$. $S A_{2} e$ leads $s_{3} \subseteq G$. Let $A_{3}=S A_{1} A_{1}, A_{3}^{\prime}=A_{1}^{\prime} A_{1}^{\prime}, A_{3}^{\prime \prime}=A_{1} A_{1}^{\prime}$ and $A_{3}^{\prime \prime \prime}=A_{1}^{\prime} A_{1}$ (see Figure 3 ).

Subcase 2.2. $\quad t_{3}=P$

It is impossible to have $P X Y$ where $X$ and $Y \subseteq\left\{e, A_{1}, A_{1}^{\prime}\right\}$ because $G$ is a simple graph. $P e A_{2}, P A_{2} e, P A_{1} A_{2}, P A_{2} A_{1}, P A_{1}^{\prime} A_{2}, P A_{2} A_{1}^{\prime}, P A_{0} A_{1}$ and $P A_{1}^{\prime} A_{0}$ lead to $s_{9} \subseteq G$. $P A_{2} A_{2}$ leads to $s_{7} \subseteq G$. Let $A_{4}=P A_{0} A_{1}^{\prime}=P A_{1} A_{0}$ and $A_{5}=P A_{0} A_{2}=P A_{2} A_{0}$ (see Figure 3 ).

Case 3. $\quad i=4$

One of the children of $t_{4}$ must be in $\left\{A_{3}, A_{3}^{\prime}, A_{3}^{\prime \prime}, A_{3}^{\prime \prime \prime}, A_{4}, A_{5}\right\}$. Since $\Delta(G)=5$, we will not consider the combinations of $A_{4}$ and $A_{4}, A_{4}$ and $A_{5}$, and, $A_{5}$ and $A_{5}$. 
Subcase 3.1. $\quad t_{4}=S$

$S X Y$ leads to $s_{2} \subseteq G$ if $X \in\left\{e, A_{0}\right\}$ and $Y \in\left\{A_{3}, A_{3}^{\prime}, A_{3}^{\prime \prime}, A_{3}^{\prime \prime \prime}\right\} . S X Y$ implies that one of $\left\{s_{6}, s_{6}^{\prime}, s_{6}^{\prime \prime}\right\}$ is a subgraph of $G$ if $X \in\left\{A_{1}, A_{1}^{\prime}, A_{3}, A_{3}^{\prime}, A_{3}^{\prime \prime}, A_{3}^{\prime \prime \prime}\right\}$ and $Y \in\left\{A_{3}, A_{3}^{\prime}, A_{3}^{\prime \prime}, A_{3}^{\prime \prime \prime}\right\} . S A_{2} Y$ leads to $s_{4} \subseteq G$ if $Y \in\left\{A_{3}, A_{3}^{\prime}, A_{3}^{\prime \prime}, A_{3}^{\prime \prime \prime}\right\} . S A_{4} Y$ leads to $s_{13} \subseteq G$ if $Y \in\left\{A_{3}, A_{3}^{\prime}\right\}$. S $A_{5} Y$ leads to $s_{10} \subseteq G$ if $Y \in\left\{A_{3}, A_{3}^{\prime}\right\}$. SA $A_{4} Y$ leads to $s_{12} \subseteq G$ if $Y \in\left\{e, A_{0}\right\}$, to $s_{13} \subseteq G$ if $Y \in\left\{A_{1}, A_{1}^{\prime}\right\}$, and to $s_{14} \subseteq G$ if $Y=A_{2} . S A_{5} Y$ leads $s_{8} \subseteq G$ if $Y \in\left\{e, A_{0}\right\}$, to $s_{10} \subseteq G$ if $Y \in\left\{A_{1}, A_{1}^{\prime}\right\}$, and to $s_{11} \subseteq G$ if $Y=A_{2}$.

Subcase 3.2. $\quad t_{4}=P$

Let $A_{6}=P A_{3} e=P e A_{3}^{\prime}, A_{6}^{\prime}=P A_{3}^{\prime} e=P e A_{3}, A_{6}^{\prime \prime}=P A^{\prime \prime} e=P e A_{3}^{\prime \prime \prime}$ and $A_{6}^{\prime \prime \prime}=P A_{3}^{\prime \prime \prime} e=P e A_{3}^{\prime \prime}$ (see Figure 3). $P A_{3} A_{0}$ and $P A_{3}^{\prime} A_{0}$ lead to $s_{25} \subseteq G$. $P A_{0} A_{3}$ and $P A_{3}^{\prime} A_{0}$ lead to $s_{24} \subseteq G$. $P X Y$ and $P Y X$ lead to $s_{24}^{\prime} \subseteq G$ if $X=A_{0}$ and $Y \in\left\{A_{3}^{\prime \prime}, A_{3}^{\prime \prime \prime}\right\} . P A_{3} A_{1}$ and $P A_{1}^{\prime} A_{3}^{\prime}$ lead to $s_{20} \subseteq G, P A_{1} A_{3}$ and $P A_{3}^{\prime} A_{1}^{\prime}$ lead to $s_{19} \subseteq G, P A_{3} A_{1}^{\prime}$ and $P A_{1} A_{3}^{\prime}$ lead to $s_{18} \subseteq G$, and $P A_{3}^{\prime} A_{1}$ and $P A_{1}^{\prime} A_{3}$ lead to $s_{21} \subseteq G$. $P X Y$ and $P Y X$ lead to either $s_{18}^{\prime} \subseteq G$ or $s_{20}^{\prime} \subseteq G$ if $X \in\left\{A_{1}, A_{1}^{\prime}\right\}$ and $Y \in\left\{A_{3}^{\prime \prime}, A_{3}^{\prime \prime \prime}\right\} . P X A_{2}$ and $P A_{2} X$ lead to $s_{4} \subseteq G$ if $X \in\left\{A_{3}, A_{3}^{\prime}, A_{3}^{\prime \prime}, A_{3}^{\prime \prime \prime}\right\}$. $P A_{3} A_{3}$ and $P A_{3}^{\prime} A_{3}^{\prime}$ lead to $s_{16} \subseteq G$. $P A_{3} A_{3}^{\prime}$ leads to $s_{15} \subseteq G$. And $P A_{3}^{\prime} A_{3}$ leads to $s_{17} \subseteq$ G. $P A_{3}^{\prime \prime} A_{3}^{\prime \prime}$ and $P A_{3}^{\prime \prime \prime} A_{3}^{\prime \prime \prime}$ lead to $s_{15}^{\prime} \subseteq$ G. $P A_{3}^{\prime \prime} A_{3}^{\prime \prime \prime}$ and $P A_{3}^{\prime \prime \prime} A_{3}^{\prime \prime}$ lead to $s_{15}^{\prime \prime} \subseteq G$. $P X A_{4}$ and $P A_{4} X$ lead to either $s_{20} \subseteq G$ or $s_{21} \subseteq G$ if $X \in\left\{A_{3}, A_{3}^{\prime}\right\} . P X A_{4}$ and $P A_{4} X$ lead to $s_{20}^{\prime} \subseteq G$ if $X \in\left\{A_{3}, A_{3}^{\prime}\right\} . P X A_{5}$ and $P A_{5} X$ lead to $s_{10} \subseteq G$ if $X \in\left\{A_{3}^{\prime \prime}, A_{3}^{\prime \prime \prime}\right\}$. It is impossible to have $P X A_{4}$ and $P A_{4} X$ where $X \in\left\{e, A_{1}\right\}$ because $G$ is a simple graph. $P A_{4} A_{2}$ and $P A_{2} A_{4}$ lead to $s_{9} \subseteq G$. $P A_{5} e$ and $P e A_{5}$ lead to $s_{9} \subseteq G$. $P A_{5} X$ and $P X A_{5}$ lead to $s_{7} \subseteq G$ if $X \in\left\{A_{0}, A_{2}\right\} . P A_{5} A_{1}$ and $P A_{1}^{\prime} A_{5}$ lead to $s_{7} \subseteq G . P A_{5} A_{1}^{\prime}$ and $P A_{1} A_{5}$ lead to $s_{9} \subseteq G$.

\section{Case 4. $\quad i=5$}

One of the children of $t_{5}$ must be in $\left\{A_{6}, A_{6}^{\prime}, A_{6}^{\prime \prime}, A_{6}^{\prime \prime \prime}\right\}$. Since $\Delta(G)=5$, we will not consider the subtrees $S X Y, P X Y$ and $P Y X$ where $X \in\left\{A_{6}, A_{6}^{\prime}, A_{6}^{\prime \prime}, A_{6}^{\prime \prime \prime}\right\}$ and $Y \in\left\{A_{4}, A_{5}, A_{6}, A_{6}^{\prime}, A_{6}^{\prime \prime}, A_{6}^{\prime \prime \prime}\right\}$.

Subcase 3.1. $t_{5}=S$

$S A_{6} Y$ leads to $s_{26} \subseteq G$ if $Y=\left\{e, A_{0}\right\}$, to either $s_{28} \subseteq G$ or $s_{30} \subseteq G$ if $Y \in\left\{A_{1}, A_{1}^{\prime}, A_{3}, A_{3}^{\prime}\right\}$, and to $s_{32} \subseteq G$ if $Y=A_{2}$. S $A_{6}^{\prime} Y$ leads to $s_{27} \subseteq G$ if $Y \in\left\{e, A_{0}\right\}$, to either $s_{29} \subseteq G$ or $s_{31} \subseteq G$ if $Y \in\left\{A_{1}, A_{1}^{\prime}, A_{3}, A_{3}^{\prime}\right\}$, and to $s_{33} \subseteq G$ if $Y=A_{2}$. $S A_{6}^{\prime \prime} Y$ and $S A_{6}^{\prime \prime \prime} Y$ lead to either $s_{26}^{\prime} \subseteq G$ or $s_{26}^{\prime \prime} \subseteq G$ if $Y \in\left\{e, A_{0}\right\}$, to either $s_{28}^{\prime} \subseteq G$ or $s_{28}^{\prime \prime} \subseteq G$ if $Y \in\left\{A_{1}, A_{3}\right\}$, to either $s_{30}^{\prime} \subseteq G$ or $s_{30}^{\prime \prime} \subseteq G$ if $Y \in\left\{A_{1}^{\prime}, A_{3}^{\prime}\right\}$, and to either $s_{32}^{\prime} \subseteq G$ or $s_{32}^{\prime \prime} \subseteq G$ if $Y=A_{2}$.

\section{Subcase 3.1. $\quad t_{5}=P$}

Since $G$ is a simple graph, we will not consider the subtrees $P X Y$ and $P Y X$ where $X \in\left\{A_{6}, A_{6}^{\prime}, A_{6}^{\prime \prime}, A_{6}^{\prime \prime \prime}\right\}$ and $Y \in\left\{e, A_{1}, A_{1}^{\prime}\right\}$.

$P A_{6} A_{0}$ and $P A_{6} A_{2}$ lead to $s_{18} \subseteq G$. $P X Y$ and $P Y X$ lead to either $s_{28} \subseteq G$ or $s_{30} \subseteq G$ if $X=A_{6}$ and $Y \in\left\{A_{3}, A_{3}^{\prime}, A_{3}^{\prime \prime}, A_{3}^{\prime \prime \prime}\right\} . P A_{0} A_{6}$ and $P A_{2} A_{6}$ lead $s_{22} \subseteq G$. $P A_{6}^{\prime} A_{0}$ and $P A_{6}^{\prime} A_{2}$ lead to $s_{19} \subseteq G$. $P X Y$ and $P Y X$ lead to either $s_{29} \subseteq G$ or $s_{31} \subseteq G$ if $X=A_{6}^{\prime}$ and $Y \in\left\{A_{3}, A_{3}^{\prime}, A_{3}^{\prime \prime}, A_{3}^{\prime \prime \prime}\right\} . P A_{0} A_{6}^{\prime}$ and $P A_{2} A_{6}^{\prime}$ lead to 
$s_{23} \subseteq G . P X Y$ leads to $s_{18}^{\prime} \subseteq G$ and $P Y X$ leads $s_{23}^{\prime} \subseteq G$ if $X \in\left\{A_{6}^{\prime \prime}, A_{6}^{\prime \prime \prime}\right\}$ and $Y \in\left\{A_{0}, A_{2}\right\} . P X Y$ and $P Y X$ lead to either $s_{30}^{\prime} \subseteq G$ or $s_{30}^{\prime \prime} \subseteq G$ if $X \in\left\{A_{6}^{\prime \prime}, A_{6}^{\prime \prime \prime}\right\}$ and $Y=A_{3}$, and to either $s_{28}^{\prime} \subseteq G$ or $s_{28}^{\prime \prime} \subseteq G$ if $X\left\{A_{6}^{\prime \prime}, A_{6}^{\prime \prime \prime}\right\}$ and $Y=A_{3}^{\prime}$. This completes the proof.

Since the algorithm we used to construct $T$ given by Takamizawa et.al. in [6] is a linear algorithm in terms of $|E(G)|$ and we traverse $T$ once to construct $T_{O}$. We may traverse the whole tree $T_{O}$ once in the worst case to find one of the 33 unavoidable subgraphs in $G$. Therefore, one can find one of the 33 unavoidable subgraphs in $G$ in $O(|E(G)|)$. This technique of constructing an oriented binary decomposition tree from any SP-graph can be applied to future research on the problems that are related to the faces and edges of any SP-graph. As an example, we will use it to prove Theorem 1 in the next section.

\section{Proof of Theorem 1}

Proof. We shall prove the theorem by induction on $|V(G)|+|E(G)|$. Clearly, the result is obvious if $|V(G)| \leq 4$. Let $G^{\prime} \subset G$ and $\phi$ be an edge-face coloring of $G^{\prime}$ where $\phi:(E F(G)) \rightarrow C=\{1,2, \ldots, 5\}$. We shall extend $\phi$ to an edge-face coloring $\sigma$ of $G$ with $t$ colors. For convenience, we denote $T(v)=C \backslash\left\{\phi\left(v v^{\prime}\right)\right.$ : $\left.v^{\prime} \in N(v)\right\}$, the set of colors missing at vertex $v$. In Figure 2 , let $f$ denote the face on the left side of $u v$ and $f^{\prime}$ denote the face on the right side of $u v$ when facing from $u$ to $v$, and, without loss of generality, let $\phi(f)=1$ and $\phi\left(f^{\prime}\right)=2$.

For $s_{1}$, let $G^{\prime}$ be the result graph by identifying vertices $x_{1}$ and $x_{2}$ in $G$. Then $\phi$ can be easily extend to $G$ since $d\left(x_{1}\right)=d\left(x_{2}\right)=2$ and $|C| \geq 5$. For $s_{2}$, let $G^{\prime}=G \backslash\left\{x_{1}\right\}$ and $c_{1} \in T(u)$. If $c_{1}=1$, then we assign $\sigma\left(u x_{2}\right)=1, \sigma\left(u x_{1}\right)=$ $2, \sigma\left(v x_{2}\right)=\sigma\left(u x_{1} x_{2}\right)=4$, and $\sigma\left(x_{1} x_{2}\right)=5$. If $c_{1} \neq 1$, we can assign $\sigma\left(u x_{2}\right)=3$, $\sigma\left(v x_{2}\right)=4, \sigma\left(u x_{1}\right)=c_{1} ; \sigma\left(x_{1} x_{2}\right)=2$ and $\sigma\left(u x_{1} x_{2}\right)=\{4,5\}-c_{1}$ if $c 1 \neq 2$ or $\sigma\left(x_{1} x_{2}\right)=5$ and $\sigma\left(u x_{1} x_{2}\right)=4$ if $c 1=2$.

For $s_{3}$, let $G^{\prime}=G \backslash\left\{x_{1}, x_{2}\right\}+u x_{3}$ and $c_{1} \in T(u)$. If $c_{1}=1$, then we assign $\sigma\left(u x_{2}\right)=1, \sigma\left(u x_{1}\right)=\sigma\left(x_{2} x_{3}\right)=3, \sigma\left(v x_{3}\right)=\sigma\left(u x_{1} x_{3} x_{2}\right)=4$ and $\sigma\left(x_{1} x_{3}\right)=5$. If $c_{1} \neq 1$, we can assign $\sigma\left(u x_{1}\right)=c_{1}, \sigma\left(x_{2} x_{3}\right)=1, \sigma\left(u x_{2}\right)=\sigma\left(x_{1} x_{3}\right)=3$, $\sigma\left(v x_{3}\right)=4$ and $\sigma\left(u x_{1} x_{3} x_{2}\right)=\{4,5\}-c_{1}$.

For $s_{4}$, let $G^{\prime}=G \backslash\left\{x_{1}, x_{2}, x_{4}\right\}+u x_{3}$. Without lose of generality, we may assume that $\phi\left(u x_{3}\right)=3$ and $\phi\left(x_{3} v\right)=4$. Let $c_{1} \in T(u)$ and $c_{2} \in T(v)$. If $c_{1}=c_{2}=1$, then we can assign $\sigma\left(u x_{2}\right)=\sigma\left(v x_{3}\right)=1, \sigma\left(x_{3} x_{4}\right)=2, \sigma\left(u x_{1}\right)=$ $\sigma\left(x_{2} x_{3}\right)=3, \sigma\left(v x_{4}\right)=\sigma\left(u x_{1} x_{3} x_{2}\right)=4$, and $\sigma\left(v x_{3} x_{4}\right)=\sigma\left(x_{1} x_{3}\right)=5$. Now consider the case where $c_{1}=1$ and $c_{2} \neq 1$. We can assign $\sigma\left(u x_{2}\right)=1, \sigma\left(u x_{1}\right)=$ $\sigma\left(x_{2} x_{3}\right)=3, \sigma\left(v x_{3}\right)=\sigma\left(u x_{1} x_{3} x_{2}\right)=4, \sigma\left(v x_{4}\right)=c_{2} ; \sigma\left(x_{1} x_{3}\right)=2, \sigma\left(x_{3} x_{4}\right)=5$ and $\sigma\left(v x_{3} x_{4}\right)=3$ if $c_{2}=2$ or $\sigma\left(x_{1} x_{3}\right)=5, \sigma\left(x_{3} x_{4}\right)=2$ and $\sigma\left(v x_{3} x_{4}\right)=\{3,5\}-$ $c_{2}$. Note that the case where $c_{1} \neq 1$ and $c_{2}=1$ can be solved similarly. Finally, we consider the case where $c_{1} \neq 1$ and $c_{2} \neq 1$. We assign $\sigma\left(u x_{1}\right)=c_{1}, \sigma\left(x_{4} v\right)=$ $c_{2}, \sigma\left(u x_{2}\right)=\sigma\left(x_{3} x_{1}\right)=3, \sigma\left(v x_{3}\right)=4, \sigma\left(x_{2} x_{3}\right)=1$, and $\sigma\left(u x_{1} x_{3} x_{2}\right) \in\{4,5\} \backslash c_{1}$; $\sigma\left(x_{3} x_{4}\right)=5, \sigma\left(v x_{3} x_{4}\right)=3$ if $c_{2}=2$ or $\sigma\left(x_{3} x_{4}\right)=2, \sigma\left(v x_{3} x_{4}\right) \in\{3,5\} \backslash c_{2}$ if $c_{2} \neq 2$. 
The rest of proof is similar with these cases. We shall leave it on the following website www.stfx.ca \people \pwang \restproof.pdf.

Remarks. The process of coloring these 33 unavoidable subgraphs takes a constant time. Hence, one can color the edges and faces of $G$ with 5 colors in $O(|E(G)|)$ time.

\section{References}

1. Borodin, O. V.: Simultaneous coloring of edges and faces of plane graphs. Discrete Math. 128 (1994) 21-33

2. Mel'nikov, L. S.: Problem in recent advances in graph theory. Proc. International Symposium, Prague, 1974, Academic Praha (1975) 543

3. Richey, M.B., Parker, R. G.: Minimum-maximal matching in series-parallel graphs. Euro. J. of Operation Research 33 (1987) 98-103

4. Sanders, D.P., Zhao, Y.: On simultaneous edge-face colorings of plane graphs. Combinatorica no. 317 (1997) 441-445

5. Seymour, P. D.: Colouring Series-Parallel graphs. Combinatoria 10(4) (1990) 379392

6. Takamizawa, K., Nishizeki, T., Saite, N.: Linear-time computability of combinatorial probelms on series-aprallel graphs. J. Assoc. Comput. Mach. 29 No. 4 (1982) 1382-1389

7. Waller, A.O.: Simultaneously coloring the edges and faces of plane graphs. J. Combinatorial Theory, Series B 69 (1997) 219-221

8. Wang, W.F.: On the colorings of outerplane graphs. Discrete Math. 147 (1995) 257-269

9. Wang, W.F., Zhang, K.M.: $\Delta$-matchings and edge-face chromatic numbers. (Chinese) Acta Math. Appl. Sinica 22(2) (1999) 236-242

10. Wang, W.F., Lih, K.W.: A new proof of Melnikov's conjecture on the edge-face coloring of plane graphs. Discrete Math. 253 (2002) 87-95

11. Wu, J.L., Wang, P.: Simutaneous coloring of edges and faces of series-parallel graphs. Adavnces in Mathematics, No.4, Vol.34 (2005) 461-467

12. Zhou, X., Suzuki, H., Nishizeki, T.: A Linear Algorithm for Edge-coloring SeriesParallel Multigraphs. J. of Algorithms 20 (1996) 174-201

13. Zhou, X., Nishizeki, T.: Multicoloring of Series-Parallel Graphs. Algorithmica 38 (2004) 271-294 